\title{
VE-LLI-VO: Vessel Enhancement Using Local Line Integrals and Variational Optimization
}

\author{
Yuan Yuan, Yishan Luo, and Albert C. S. Chung
}

\begin{abstract}
Vessel enhancement is a primary preprocessing step for vessel segmentation and visualization of vasculatures. In this paper, a new vessel enhancement technique is proposed in order to produce accurate vesselness measures and vessel direction estimations that are less subject to local intensity abnormalities. The proposed method is called vessel enhancement using local line integrals and variational optimization (VE - LLI - VO). First, vessel enhancement using local line integrals (VE - LLI) is introduced in which a vessel model is embedded by regarding a vessel segment as a straight line based upon the second order information of the local line integrals. Useful quantities similar to the eigenvalues and eigenvectors of the Hessian matrix are produced. Moreover, based upon the local line integrals, junctions can be detected and handled effectively. This can help deal with the bifurcation suppression problem which exists in the Hessian-based enhancement methods. Then a more generic curve model is embedded to model vessels and a variational optimization (VO) framework is introduced to generate optimized vesselness measures. Experiments have been conducted on both synthetic images and retinal images. It is experimentally demonstrated that VE - LLI - VO produces improved performance as compared with the widely used techniques in terms of both vesselness measurement and vessel direction estimation.
\end{abstract}

Index Terms-Retinal images, vessel enhancement.

\section{INTRODUCTION}

$\mathbf{O}$ PTIC FUNDUS photography technique is a widely used procedure for the diagnosis of retinopathies. The inspection of vasculatures in the retinal images not only plays an important role in diagnosing ophthalmic diseases, e.g., retinal artery occlusion, glaucoma, but also helps reveal nonophthalmic diseases, such as hypertension, diabetes, arteriosclerosis, cardiovascular disease and stroke. However, the current optic fundus photographs often present remarkable noise, inadequate intensity contrast and intensity variations. These effects make the analysis of the retinal images difficult. To improve the retinal image quality, vessel enhancement, which is primarily used as a preprocessing step for vessel

Manuscript received July 28, 2009; revised March 01, 2010 and August 25, 2010; accepted November 15, 2010. Date of publication December 06, 2010; date of current version June 17, 2011. This work was supported by the K S Lo Foundation, Hong Kong. The associate editor coordinating the review of this manuscript and approving it for publication was Prof. Scott T. Acton.

The authors are with Lo Kwee-Seong Medical Image Analysis Laboratory, Department of Computer Science and Engineering, The Hong Kong University of Science and Technology, Hong Kong (e-mail: yaleyuan@gmail.com; lisaluo@cse.ust.hk; achung@cse.ust.hk).

Color versions of one or more of the figures in this paper are available online at http://ieeexplore.ieee.org.

Digital Object Identifier 10.1109/TIP.2010.2097269 segmentation and visualization, has been studied intensively by different research groups in the past decades.

Vessel enhancement methods often take an image as input and for each point, a vesselness measure is calculated based upon local image features. Among all the vessel enhancement methods, the Hessian-based methods have been used widely because of the fact that its core component, the Hessian matrix, offers elegant geometrical interpretations and is able to retrieve useful vascular information from the images. In this work, the Hessian-based methods refer to those techniques that exploit the eigenvalues and/or the eigenvectors of the Hessian matrix during the vessel enhancement procedure. To the best of our knowledge, Koller et al. [1] were the first research group to propose the use of information extracted from the Hessian matrix in the context of vessel enhancement. Their method uses the eigenvectors of the Hessian matrix for vessel direction estimation and applies a matching filter along the vessel direction to detect vessel presence. Krissian et al. [2] later refined this matching filter. While only the eigenvectors of the Hessian matrix are used in [1] and [2], other works [3]-[6] take the eigenvalues into consideration. In these works, the eigenvalues are combined to distinguish curve-linear structures from the image background region. Among the previous methods, a method proposed by Frangi et al. [4] is one of the most commonly used vessel enhancement methods in practice. It utilizes all the eigenvalues and has an intuitive geometric interpretation.

While the Hessian-based methods are widely used in different areas of image processing [7]-[11], its drawback is obvious due to the fact that the Hessian matrix is based upon second derivatives, which are local measures. Therefore, without a relatively global view of the vasculatures, the Hessian-based methods only offer local shape descriptions. It, hence, suffers from over sensitivity to local intensity abnormalities, which can be viewed as deviations of intensity structures from the targeted ones, e.g., 1) noise which gives false positive responses; 2) uneven responses inside a vessel; and 3) suppressed bifurcations. This property deteriorates the effect of visualization, and also adversely affects the quality of the subsequent segmentation methods, especially for variational segmentation frameworks such as [12] in which the contour evolution can get stuck at local optimum.

In all the previous works, a vesselness measure reflects the possibility that a point belongs to a vessel. Each image point is therefore viewed as an individual and only information inside the local region of this point is used to compute the vesselness responses. It does not take into account the geometrical property of the particular vessel segment to which a point belongs. Not only should a more robust vesselness measure reflect whether a point belongs to a vessel segment, but also reveal whether the 
vessel segment is a strong one or not. As will be described in this paper, a new view on vesselness measure should shift its focus from a point to the vessel segment in which the point resides, and make the measure less sensitive to local intensity abnormalities. To that attempt, vessel enhancement using local line integrals and variational optimization $(\mathbf{V E}-\mathbf{L L I}-\mathbf{V O})$ is proposed in this paper. In the first step, vessel enhancement using local line integrals $(\mathbf{V E}-\mathbf{L L I})$ is introduced. It models a vessel segment as a straight line and exploits the second order information of the local line integrals in order to capture potentially existing line structures with less sensitivity to local intensity abnormalities. Useful quantities similar to the eigenvalues and eigenvectors of the Hessian matrix are produced in a multiscale fashion and then combined to generate a vesselness measure. With the new vesselness measure, it is relatively easy to detect junction points in the images. With an adaptive length for line integrals, junction regions can be detected and enhanced. By using this straight line vessel model, VE - LLI is able to take more information into consideration and can give better vessel direction estimations and vesselness responses, as will be demonstrated in the experiments.

To further improve the quality of the vesselness measures, a variational optimization ( VO) framework is proposed to extend $\mathbf{V E}$ - LLI to form the VE - LLI - VO method. A more generic curve model is used to model vessel segments to capture vessels with various shapes, in contrast to the straight line model used in the previous VE - LLI. VE - LLI - VO calculates an optimized vesselness measure based upon the features of the possible vessel segment (more specifically, the centerline of the vessel segment) to which a point belongs. VE - LLI - VO can produce smooth vesselness responses along vessels and give good performance in background suppression. The new method has been tested on both synthetic and retinal images. Detailed quantitative and qualitative comparisons will be presented in Section VI.

This paper is organized as follows. In Section II, three Hessian-based vessel enhancement methods are briefly introduced and compared. Section III gives a full account of VE - LLI. In order that the later experiments are fair, optimal parameters are searched (see Section IV) based upon the analysis of enhancement results. The idea of VE - LLI - VO is elaborated in Section V. The underlying principle of VE - LLI - VO is described with illustrations. In Section VI, VE - LLI - VO is tested on synthetic images and two publicly available databases of the retinal images. Qualitative and quantitative results obtained by using different methods are presented and discussed. This paper is concluded in Section VII.

\section{Hessian-Based Enhancement Methods}

Given a $\mathcal{N}$-dimensional image $I: \Omega \rightarrow \mathbb{R}, \Omega \subseteq \mathbb{R}^{\mathcal{N}}$, the Hessian matrix of $I$ at point $x$ is defined as

$$
H(x)=\left[\begin{array}{cccc}
\frac{\partial^{2} I}{\partial x_{1} \partial x_{1}} & \frac{\partial^{2} I}{\partial x_{1} \partial x_{2}} & \cdots & \frac{\partial^{2} I}{\partial x_{1} \partial x_{\mathcal{N}}} \\
\frac{\partial^{2} I}{\partial x_{2} \partial x_{1}} & \frac{\partial^{2} I}{\partial x_{2}^{2}} & \cdots & \frac{\partial^{2} I}{\partial x_{2} \partial x_{\mathcal{N}}} \\
\vdots & \vdots & \ddots & \vdots \\
\frac{\partial^{2} I}{\partial x_{\mathcal{N}} \partial x_{1}} & \frac{\partial^{2} I}{\partial x_{\mathcal{N}} \partial x_{2}} & \cdots & \frac{\partial^{2} I}{\partial x_{\mathcal{N}} \partial x_{\mathcal{N}}}
\end{array}\right]
$$

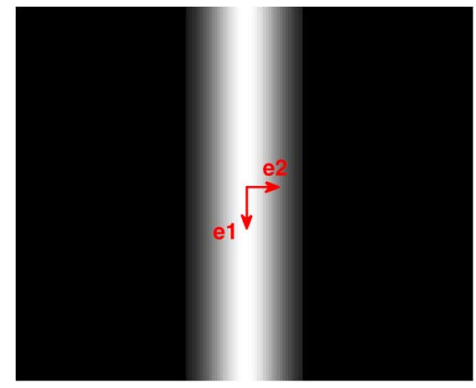

Fig. 1. Synthetic vessel with Gaussian intensity profile, together with the eigenvectors shown at the center.

TABLE I

EIGENVALUES FOR DiFFERENT 2-D STRUCTURES, WiTH ORDERING $\left|\lambda_{1}\right| \leq\left|\lambda_{2}\right|$. “- - "Means Negative Quantity With LaRge Magnitude, While “ $\approx 0$ ” MeAns Near Zero Value

\begin{tabular}{|c|c|c|}
\hline & Vessel & Background \\
\hline \hline$\lambda_{1}$ & $\approx 0$ & $\approx 0$ \\
\hline$\lambda_{2}$ & -- & $\approx 0$ \\
\hline
\end{tabular}

where $x_{i}, i \in\{1,2, \ldots, \mathcal{N}\}$, are variables for $I$. Eigenvectors $e_{i}$ and the corresponding eigenvalues $\lambda_{i}$ are important shape descriptors for $I$. In 2-D case, $e_{1}\left(e_{2}\right)$ gives the direction along which the surface has minimum(maximum) curvature, with $\lambda_{1}\left(\lambda_{2}\right)$ being the corresponding eigenvalue. In this work, vessels are considered brighter than the background; otherwise, the image is negated. Fig. 1 shows a 2-D synthetic vessel with a Gaussian intensity profile. The eigenvectors of the Hessian matrix at the center are shown. It can be seen that the surface does not curve along the vessel direction and therefore has a curvature of zero. While along the normal direction of the vessel, the surface is curved and, hence, has a negative curvature with large magnitude.

Suppose that the ordering of the eigenvalues is $\left|\lambda_{1}\right| \leq\left|\lambda_{2}\right|$. The eigenvalues for different structures can then be summarized in Table I. A " - - " sign means that the quantity is negative with large magnitude, while a " $\approx 0$ " sign means that the value is near zero.

Using the eigenvalues and/or eigenvectors of the Hessian matrix, different groups of researchers have designed a wide spectrum of vessel enhancement techniques. Three widely studied methods of this kind are Krissian's method [2], Li's method [5] and Frangi's method [4]. In all three methods, the Gaussian models are exploited for modeling the intensity profile on the vessel cross-section. Suppose that $y$ axis is the vessel axis for 2 -D vessel, the intensity on the vessel cross-section is modeled as

$$
I(x, y)=C e^{-\frac{x^{2}}{2 \sigma_{s}^{2}}} .
$$

$C$ is a normalizing constant equal to the maximum intensity in the cross-section while $\sigma_{s}$ is the scale of the Gaussian model. Vessel boundaries are considered as points that maximize the gradient magnitude, therefore, at $x=\sigma_{s}$. The vessel radius is then $\sigma_{s}$. Hereinafter, the ordering of the eigenvalues and the eigenvectors of the Hessian matrix is $\left|\lambda_{i}\right| \leq\left|\lambda_{j}\right|$ if $i<j$, with 
the corresponding unit eigenvectors $e_{i}$ and $e_{j}$, unless specified otherwise.

\section{A. Krissian's Vesselness Measure}

Based upon the work of [1], Krissian et al. [2] proposed a vessel enhancement filter relying on the eigenvectors of the Hessian matrix and the image gradients. The local maxima of the filter response are used to find the center points and the radius information so that a cylindrical model is fit to the observed data. For 3-D, the response of scale $\sigma_{s}$ at $\mathbf{x}$ is given as

$$
K_{\sigma_{s}}^{3-D}(\mathbf{x})=\frac{1}{N} \sum_{i=0}^{N-1}\left\langle-\nabla I\left(\mathbf{x}+\sigma_{s} \vec{v}_{\alpha}\right), \vec{v}_{\alpha}\right\rangle
$$

with $\alpha=2 \pi i / N, \vec{v}_{\alpha}=\cos (\alpha) e_{2}+\sin (\alpha) e_{3}, e_{2}$ and $e_{3}$ are the eigenvectors of the Hessian matrix $H(\mathbf{x})$ which form an orthogonal basis on the vessel cross section. While Krissian et al. do not provide a 2-D equivalent formulation, a 2-D version can be derived with $N=2$ in the (3) as

$K_{\sigma_{s}}^{2-D}(\mathbf{x})=\frac{1}{2}\left(\left\langle-\nabla I\left(\mathbf{x}+\sigma_{s} e_{2}\right), e_{2}\right\rangle+\left\langle\nabla I\left(\mathbf{x}-\sigma_{s} e_{2}\right), e_{2}\right\rangle\right)$.

Krissian's measure only uses the eigenvectors of the Hessian matrix as vessel frame guidance and uses the first order information (i.e., image gradient). The measure depends upon the vessel-background intensity contrast in the particular region being enhanced. Moreover, it tends to suppress junctions since junctions cannot be characterized as a cylindrical model.

\section{B. Li’s Vesselness Measure}

In [5], through the eigenvalue analysis of the Hessian matrix, three selective enhancement filters for dot, line and plane are proposed which can simultaneously enhance objects of specific shapes and suppress objects of other shapes. To extract a vessel, a line enhancement filter is employed by combining a likelihood function and a magnitude function based upon the eigenvalues of the Hessian matrix. The likelihood function indicates probability that a pixel belongs to a line, and the magnitude function indicates the existence of a line exist in a background. A vesselness response $L_{\sigma_{s}}^{2-D}(\mathbf{x})$ at point $\mathbf{x}$ of scale $\sigma_{s}$ is given by

$$
L_{\sigma_{s}}^{2-D}(\mathbf{x})= \begin{cases}0, & \text { if } \lambda_{2} \geq 0 \\ \left|\lambda_{2}\right|-\left|\lambda_{1}\right|, & \text { otherwise. }\end{cases}
$$

The method uses all the eigenvalues of the Hessian matrix and does not use the eigenvectors. As compared with the Krissian's measure, Li's measure does not depend upon vessel-background intensity contrast but on the shape of local intensity surface. However, noise is a potential major factor affecting the performance of Li's measure, as the measure uses the second derivatives which are inherently sensitive to noise.

\section{Frangi's Vesselness Measure}

Frangi et al. [4] proposed a multiscale vesselness measure which assigns a value between 0 and 1 to each point in the image. This assigned value reflects the confidence of a point being inside a vessel. For a single scale $\sigma_{s}$, a response at a point $\mathbf{x}$ is calculated as

$$
F_{\sigma_{s}}^{2-D}(\mathbf{x})= \begin{cases}0, & \text { if } \lambda_{2} \geq 0 \\ e^{-\frac{R_{b}^{2}}{2 \beta^{2}}}\left(1-e^{-\frac{S^{2}}{2 c^{2}}}\right), & \text { otherwise }\end{cases}
$$

where $R_{b}=\left|\lambda_{1}\right| /\left|\lambda_{2}\right|$ and $S=\left(\lambda_{1}^{2}+\lambda_{2}^{2}\right)^{1 / 2}$, and $\beta, c$ are constant normalization factors. $R_{b}$ is essential for distinguishing between plate-like and line-like structures since only in the latter case it becomes zero. $S$ is a measure of the "second order structureness." The value of $S$ becomes low in the background where no structure is present and the eigenvalues are small for the lack of intensity contrast. The underlying principle behind the Frangi's filter is to measure the vesselness according to the two criteria expressed by $R_{b}$ and $S$. The filter contains two parameters for 2-D, which are suggested by Frangi et al. to set $\beta=0.5$ and $c=(1 / 2) \max \{S(x) \mid x \in \Omega\}$.

\section{Multiscale Processing}

In all of the previously mentioned vessel enhancement methods, the concept of the normalized derivatives proposed by Lindeberg [13] is used to deal with multiscale normalization. Numerical differentiation of an image at a scale $\sigma_{s}$ is defined as

$$
\left(\frac{\partial}{\partial x_{i}}\right)_{\sigma_{s}} I \triangleq \sigma_{s}^{\gamma} I * \frac{\partial}{\partial x_{i}} G_{\sigma_{s}}
$$

where $\gamma$ is a normalizing term, and $G_{\sigma_{s}}$ is the Gaussian smoothing function. Lindeberg suggested that if there is no preference of vessels with particular width, $\gamma$ should be set to 1. It is worth to mention that the image at each scale is first convolved with a Gaussian smoothing function before calculating the second derivatives. Gaussian blurring can reduce the influence of noise and properly shape the intensity on the vessel cross section into a Gaussian profile, so as to ensure a large value of second derivative across the vessel cross section, even in the case of a plateau-like profile or even a slight intensity dip at the line center.

Given a set of scales $\Omega_{\sigma_{s}}$, responses of different scales are combined as

$$
E(\mathbf{x})=\max _{\sigma_{s}}\left\{E_{\sigma_{s}}(\mathbf{x}) \mid \sigma_{s} \in \Omega_{\sigma_{s}}\right\}
$$

Also $\vec{V}(\mathbf{x})=e_{1}^{*}$ is the estimation of vessel direction, where $e_{1}^{*}$ is the eigenvector corresponding to the optimal scale. The set of scales is usually chosen to cover the range of vessel radii in the image region.

\section{E. Comparison of Enhancement Results}

The three enhancement techniques have been applied to 2-D retinal images. Fig. 2 shows a comparison between the enhancement results obtained by using the three methods. In Fig. 2, images are organized from left to right as follows: the original image, the ground truth, the results obtained by using the Krissian's, Li's and Frangi's methods, respectively. In Frangi's measure, $\beta=0.5$ and $c=(1 / 2) \max \{S(x) \mid x \in \Omega\}$ as suggested 

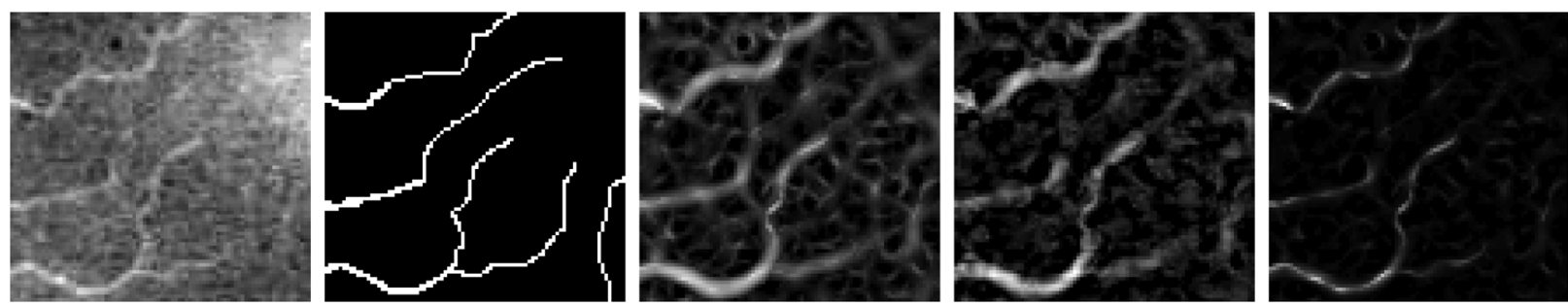

Fig. 2. Comparison of enhancement results. From left to right, a noisy retinal image, its ground truth, enhancement results by using Krissian's, Li's and Frangi's methods, respectively. (Please refer to the electronic version for better visualization.)

by Frangi et al. [4]. Six scales were used with $\sigma_{\min }=1$ and $\sigma_{\max }=3$.

From the figure, the following is observed.

- All methods can distinguish vessels and achieve different levels of background suppression.

- Frangi's method has a better background suppression performance than the other two methods, especially at noisy regions (Fig. 2). In general, Krissian's method does not perform well in recognizing vessels in low contrast and noisy background regions due to its use of first derivatives instead of second order information.

- Responses produced by using Frangi's and Li's methods tend to be discontinuous inside and along vessels in noisy regions.

With a far better background suppression performance and the flexibility of changing parameters, Frangi's method is generally considered the most satisfactory among the three methods. In the next section, we will present a method that will give reliable responses inside vessels and meanwhile suppress noise in the background regions.

\section{Vessel ENhancement Using Local Line InTEgRalS}

The Hessian matrix encodes useful information in a compact way and those information can be easily retrieved via the eigen-decomposition. However, as the Hessian matrix is a local quantity, any Hessian matrix based measures depending upon the eigenvalues and the eigenvectors will be sensitive to local intensity structures and also intensity abnormalities, e.g., noise which can give false positive results, and discontinuous responses along vessel direction. These effects are undesirable for both vessel enhancement and visualization improvement.

Since a more robust vesselness measure should not only predict whether a point belongs to a vessel segment, but also reflect whether the vessel segment is a strong one or not. It will be useful to develop quantities based upon the information extracted from the vessel segment to which a point belongs, instead of the point itself. A vesselness measure can be calculated based upon these quantities in order to give smooth and reliable responses inside vessels meanwhile suppress noise in the background. It will also be very useful if the newly developed quantities can be embedded in any existing enhancement framework.

In this section, vessel enhancement using local line integrals (VE - LLI) is introduced in order to achieve these goals. The idea is to model vessels by using straight line segments and extract information from the local line integrals. By regarding a vessel segment as a straight line, quantities similar to eigenvalues and eigenvectors of the Hessian matrix can be computed from the local line integrals of the Hessian matrix. These quantities are more robust to local abnormalities and, hence, can capture a larger and better view on the presence of vessels.

\section{A. Local Line Integrals}

Given a $\mathcal{N}$-dimensional image $I: \Omega \rightarrow \mathbb{R}, \Omega \in \mathbb{R}^{\mathcal{N}}$, a local line integral transform can be defined as

$$
R^{\vec{v}}[I](\mathbf{x})=\frac{1}{d} \int_{-\frac{d}{2}}^{\frac{d}{2}} I\left(L_{\vec{v}}(t)\right) d t
$$

where $L_{\vec{v}}(t)=\mathbf{x}+t \vec{v}$ is a line parameterized by $t$. Unit vector $\vec{v}$ represents the line direction, $d$ is the integral length and $1 / d$ is a normalizing term. While $R^{\vec{v}}[I]$ gives the intensity average of $I$ along a local line, partial derivative of $R^{\vec{v}}[I]$ gives the average of partial derivatives of $I$. In particular, for the second derivatives

$$
\frac{\partial^{2}}{\partial x_{i} \partial x_{j}} R^{\vec{v}}[I](\mathbf{x})=\frac{1}{d} \int_{-\frac{d}{2}}^{\frac{d}{2}} \frac{\partial^{2}}{\partial x_{i} \partial x_{j}} I\left(L_{\vec{v}}(t)\right) d t
$$

where $x_{i}$ and $x_{j},\{i, j\} \in\{1,2, \ldots, \mathcal{N}\}$, are variables for both $I$ and $R^{\vec{v}}$.

Let $H(\mathbf{x})$ be the Hessian matrix for $I$ at point $\mathbf{x}$. Following (1) and (10), the Hessian matrix $\bar{H}^{\vec{v}}(\mathbf{x})$ for $R^{\vec{v}}[I]$ at point $\mathbf{x}$ is then

$$
\bar{H}^{\vec{v}}(\mathbf{x})=\frac{1}{d} \int_{-\frac{d}{2}}^{\frac{d}{2}} H\left(L_{\vec{v}}(t)\right) d t .
$$

Eigenvectors $\bar{e}_{i}^{\vec{v}}$ and the corresponding eigenvalues $\bar{\lambda}_{i}^{\vec{v}}$, where $\left|\bar{\lambda}_{1}^{\vec{v}}\right| \leq\left|\bar{\lambda}_{2}^{\vec{v}}\right| \leq \cdots \leq\left|\bar{\lambda}_{\mathcal{N}}^{\vec{v}}\right|, i \in\{1,2, \ldots, \mathcal{N}\}$, of $\bar{H}^{\vec{v}}$ give interesting measures of structures along the local line. Notice that $\bar{e}_{i}^{\vec{v}}$ and $\bar{\lambda}_{i}^{\vec{v}}$ are functions of $\vec{v}$. Consider $\mathcal{N}=2$. Let $\lambda_{1}$, $\lambda_{2}\left(\left|\lambda_{1}\right| \leq\left|\lambda_{2}\right|\right)$ be the eigenvalues of $H(\mathbf{x})$ at the point $\mathbf{x}$ and $e_{1}, e_{2}$ be the corresponding eigenvectors. As summarized in Table I, $\left|\lambda_{1}\right| \ll\left|\lambda_{2}\right|$, along with $\lambda_{1} \approx 0, \lambda_{2} \ll 0$, these conditions signal a likely presence of vessel structure at point $\mathbf{x}$. Furthermore, $e_{1}$ gives an estimation of the vessel direction.

In order to find the probing line that can best approximate the vessel segment, it is observed that, at a vessel point $\mathbf{x}$, when the integral line $L_{\vec{v}}(t)$ is aligned with the vessel, it will also have the following relationships $\left|\vec{\lambda}_{1}^{v}\right| \ll\left|\bar{\lambda}_{2}^{\vec{v}}\right|, \bar{\lambda}_{1}^{\vec{v}} \approx 0, \bar{\lambda}_{2}^{\vec{v}} \ll 0$, which 
are the same to the relationships as listed previously. ${ }^{1}$ At the vessel point, $\bar{H}^{\vec{v}}$ becomes the average of the similar Hessian matrices along $L_{\vec{v}}(t)$ according to (11), given the assumption that the intensity variations are relatively small along the vessel. By replacing the Hessian matrix of each pixel along the line with the average Hessian matrix defined in (11), it can result in smoothed Hessian matrices with the reduction in sharp transitions across matrices because smoothing Hessian matrices is equivalent to smoothing the second partial derivatives of the image. As noise can cause the sharp transitions in Hessian matrices, thus, this averaging process makes $\mathbf{V E}$ - LLI more resistant to noise. This smoothing method is superior to the Gaussian smoothing used by the other Hessian-based methods as it smoothes the signal along the probing line $L_{\vec{v}}(t)$ rather than in arbitrary directions and, thus, the vessel boundary can be better preserved. Furthermore, $\vec{e}_{1}^{\vec{v}}$ will have the same direction as $\vec{v}\left(\vec{e}_{1}^{\vec{v}}= \pm \vec{v}\right)$, the direction of the integral line. Therefore, when $L_{\vec{v}}(t)$ and the vessel are aligned at a vessel point $\mathbf{x},\left|\bar{\lambda}_{2}^{\vec{v}}\left\langle\vec{v}, \bar{e}_{1}^{\vec{v}}\right\rangle\right|$ will be maximized. An estimation of vessel direction can be obtained when $\left|\bar{\lambda}_{2}^{\vec{v}}\left\langle\vec{v}, \bar{e}_{1}^{\vec{v}}\right\rangle\right|$ is maximized. We choose

$$
\vec{v}^{*}=\arg \max _{\vec{v}}\left|\bar{\lambda}_{2}^{\vec{v}}\left\langle\vec{v}, \bar{e}_{1}^{\vec{v}}\right\rangle\right|
$$

as the best probing direction. Let $\bar{\lambda}_{i}^{*}=\bar{\lambda}_{i}^{\vec{v}^{*}}$ and $\bar{e}_{i}^{*}=\bar{e}_{i}^{*}$ be the eigenvalues and the eigenvectors of $\bar{H}^{\vec{v}^{*}}$, respectively. These quantities can then be used in any Hessian-based measures by simply replacing the corresponding values. In addition, notice that in order to obtain good estimations, $\vec{v}$ has to cover the half unit sphere in $\mathbb{R}^{\mathcal{N}}$ uniformly. In all the 2-D experiments in this work, $\vec{v}=[\cos \theta, \sin \theta]$, where $\theta=\left\{10^{\circ}, 20^{\circ}, \ldots, 180^{\circ}\right\}$.

\section{B. Vesselness Measure}

Though it should be clear that the new $\bar{e}_{i}^{*}$ and $\bar{\lambda}_{i}^{*}$ can be used in any Hessian-based vesselness measures, Frangi's measure in (6) is used to combine $\bar{\lambda}_{i}^{*} \mathrm{~s}$ in VE - LLI, with multiscale analysis. The reason for choosing Frangi's measure is that it gives better performance than the other two methods considered in this work. Also, since Frangi's measure only uses eigenvalues, only improvements on $\bar{\lambda}_{i}^{*}$ can be shown by directly comparing enhancement results. Improvements on $\bar{e}_{i}^{*}$ will also be analyzed in the experiments section in Section VI through comparisons on vessel direction estimations.

\section{Line Integral Length $d$}

By probing all local lines of length $d, \mathbf{V E}-\mathbf{L L I}$ can determine whether a point belongs to a vessel based upon more information than mere local intensity structures. Responses produced by VE - LLI can reflect whether a point belongs to a vessel and whether the vessel segment is strong or not. The choice of $d$ depends upon image resolution and curvatures of vessels in the

\footnotetext{
${ }^{1}$ At a vessel point $\mathbf{x}$, when the probing line $L_{\vec{v}}(t)$ is aligned with the vessel, $H\left(L_{\vec{v}}(t)\right)$ for each pixel along the line segment will have eigenvector $e_{1}$ pointing to a direction parallel to the vessel direction under the assumption that the intensity variations are relatively small along the vessel. With identical eigenvectors for each $H\left(L_{\vec{v}}(t)\right)$, the eigenvalue $\bar{\lambda}_{1}^{\vec{v}}\left(\bar{\lambda}_{2}^{\vec{v}}\right)$ of $\bar{H}^{\vec{v}}(\mathbf{x})$ is equal to the sum of the eigenvalues $\lambda_{1}\left(\lambda_{2}\right)$ of individual $H\left(L_{\vec{v}}(t)\right)$. Therefore, the relationship between $\lambda_{1}$ and $\lambda_{2}$ will be preserved between $\bar{\lambda}_{1}^{\vec{v}}$ and $\bar{\lambda}_{2}^{\vec{v}}$.
}

image. If the value of $d$ is too large, responses at vessel structures with high curvature will decline. On the other hand, if the value of $d$ is too small, noise will not be effectively reduced.

\section{Junction Region Processing}

One limitation of the Hessian-based methods is that it is difficult to obtain accurate enhancement results at junctions, as it takes a local view to check whether a point belongs to a vessel segment or not. Using VE - LLI, it not only can detect the junctions in the vessel images, but also can give better enhancement results in these junction regions with certain adjustment of the line integral length $d$. Here junction region is defined as the local neighboring region containing a vessel point where vessels of different directions meet, including bifurcation and crossing.

From the construction of local line integrals in Section III-A, it is observed that if only one vessel passes through the vessel point, the values of $\left|\vec{\lambda}_{2}^{\vec{v}}\left\langle\vec{v}, \bar{e}_{1}^{\vec{v}}\right\rangle\right|$ with respect to the sampling angles (relative the vertical direction) at the vessel point contain only one obvious peak, as there is only one optimal estimated direction for $\vec{v}$ that matches $\bar{e}_{1}^{\vec{v}}$. However, if several vessels with different directions meet at the vessel point, it will present several obvious peaks. Each peak corresponds to one estimated vessel direction. This inherent property of VE - LLI model can help find the junctions in vessel images. Therefore, the peaks of $\left|\vec{\lambda}_{2}^{\vec{v}}\left\langle\vec{v}, \bar{e}_{1}^{\vec{v}}\right\rangle\right|$ with respect to the sampling angles are first detected. The peaks are detected by the peaks finding algorithm proposed in [14]. The algorithm detects peaks by looking for downward zero-crossings in the smoothed first derivatives that exceed SlopeThreshold and peak amplitudes that exceed AmpThreshold. The two parameters, SlopeThreshold and AmpThreshold, are respectively set to $0.5 \times$ WidthPoints $^{-2}$ and $0.05 \times \max \{$ signal amplitude $\}$ (i.e., $0.05 \times \max \left\{\left|\vec{\lambda}_{2}^{\vec{v}}\left\langle\vec{v}, \vec{e}_{1}^{\vec{v}}\right\rangle\right|\right\}$ in this paper) as suggested in [14]. The parameter WidthPoints is the average number of points within the half-width of a peak (i.e., the peak width at half of the peak height), which is set to four points in our experiments.

Through applying the peaks finding algorithm, if more than one peak are detected at a vessel point, the point is regarded as a junction point. After detecting the junction, a smaller line integral length $d^{\prime}$ (i.e., $\lceil 0.5 d\rceil$ ) is chosen to process the local region of the junction point (i.e., $d^{\prime} \times d^{\prime}$ region centered at the junction point) so that the response in the junction region can be more accurate. Fig. 3 shows the junction region detection in a retina image. The green and red dots show two junction regions, while bule circle marks a single-direction vessel point. With integral length $d=13$, the values of $\left|\bar{\lambda}_{2}^{\vec{v}}\left\langle\vec{v}, \bar{e}_{1}^{\vec{v}}\right\rangle\right|$ with respect to the sampling angles $\left\{10^{\circ}, 20^{\circ}, \ldots, 180^{\circ}\right\}$ for the three vessel points are plotted in Fig. 3(b)-(d) respectively. It can be observed that Fig. 3(b) and (c) show more than one distinct peaks, which indicate the two points belong to junction regions.

\section{PARAMETER OPTIMIZATION}

As VE - LLI is based upon the vesselness measure proposed by Frangi et al., parameters in Frangi's vesselness measure need to be optimized before any quantitative comparisons. Since detailed performance evaluations will be conducted on 2-D retinal images obtained from the DRIVE [15], [16] and 


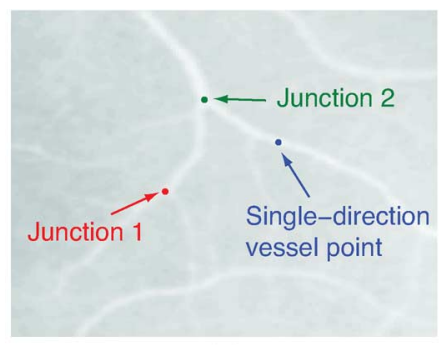

(a)

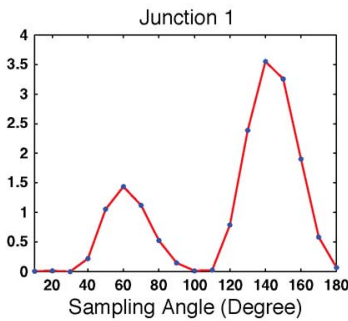

(b)

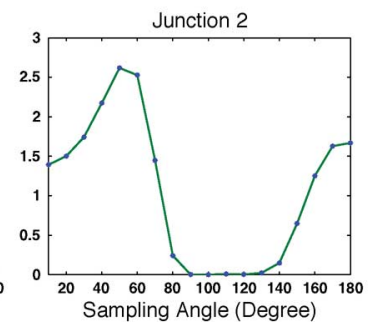

(c)

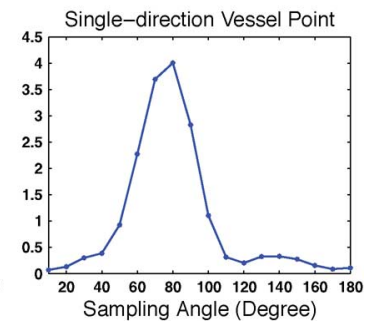

(d)

Fig. 3. Junction region detection in a 2-D retina image. (a) shows a 2-D retina image. Red and green dots indicate the junction regions and blue circle indicates the single-direction vessel point. The values of $\left|\bar{\lambda}_{2}^{\vec{v}}\left\langle\vec{v}, \bar{e}_{1}^{\vec{v}}\right\rangle\right|$ with respect to the sampling angles for the three points are shown respectively in (b), (c), and (d), where more than one peak pattern can be observed in the curves for the junction points. In (b), (c), and (d), the x-axes start from 10 to $180^{\circ}$.

STARE [17] databases, an introduction to the two databases will be first given in this section. Following the introduction, methods for optimizing parameters are described.

\section{A. DRIVE and STARE Databases}

Both DRIVE and STARE are publicly available 2-D retinal image databases. The DRIVE database was collected by Niemeijer et al. and consists of 40 images. The images are divided into two groups, namely, the training set and the testing set, containing 20 retinal images each. The training set is useful for parameter training in the segmentation and enhancement methods; while the testing set is usually used for method validation. Each of the 40 images in DRIVE has a corresponding binary mask which delineates the circular field of view (FOV) in the image. The testing set also provides two sets of manually segmented vasculatures, in which, the first set is normally used as the ground truth. In contrast, there is one set of segmented results for the training set. Each image in DRIVE is of size 565 by 584 pixels and the $45^{\circ} \mathrm{FOV}$ is circular with a diameter of approximately 540 pixels.

The STARE database was collected by Hoover et al. It consists of 20 images, each of size 700 by 605 pixels. The $35^{\circ} \mathrm{FOV}$ in the images are approximately of size 650 by 550 pixels. For each image, two sets of segmented results are provided. Similar to DRIVE, the first set of observations is used as the ground truth.

In order to find the pixel size relationship between images from DRIVE and STARE, we assume that, when capturing retinal images, the distance $h$ from the camera optical center to the retinal surface remains the same. Let $U_{D}$ and $U_{S}$ be the corresponding pixel sizes for DRIVE and STARE, respectively. It should have $U_{D}(540 / 2) / h=\tan \left(45^{\circ} / 2\right)$ and $U_{S}(650 / 2) / h=\tan \left(35^{\circ} / 2\right)$. Therefore, $U_{D} / U_{S} \approx 1.58$. Because of this relationship, and the same target objects obtained by using the same imaging modality in the two databases, integral length $d_{D}$ for DRIVE and $d_{S}$ for STARE can be related by $1.58 d_{D}=d_{S}$.

\section{B. Optimization of Parameters for Frangi's Vesselness Measure}

For Frangi's vesselness measure, $\beta, c$ are parameters, whose default values are $\beta=1 / 2$ and $c=(1 / 2) \max \{S(x) \mid x \in \Omega\}$. Denote $c$ as $c=k \max \{S(x) \mid x \in \Omega\}$. The default setting of parameters suffices to produce enhancement results of quality for a wide range of 2-D and 3-D images. Due to the differences in the nature of the imaging technique and the type of vasculatures, optimal parameters can be different for each type of images. In all fairness, it is desirable to search for an optimal set of parameters before any quantitative experiments are conducted.

Since experiments in Section VI will be conducted on 2-D retinal images from DRIVE and STARE, optimal values of $\beta$ and $k$ are first searched based upon the enhancement results of the images from these databases. Furthermore, because of the same target objects in both DRIVE and STARE which are obtained by using the same imaging modality, and the deduced relationship between $d_{D}$ and $d_{S}$, the search of optimal parameters can be done in one database for the efficiency concern. In the current study, the DRIVE database is chosen for the training purpose because of its provided masks and the fact that it contains fewer images with pathologies, which are likely to affect the process.

Parameters are evaluated by using the receiver operating characteristic (ROC) curves on the enhanced results obtained by using the Frangi's vesselness measure stated in (6) with the particular parameter setting. ROC curves are plots of false positive ratios (FPR) on the $x$-axis against true positive ratios (TPR) on the $y$-axis. It is an important benchmark usually exploited for evaluating segmentation methods. Each segmented result corresponds to a point on the ROC curve. Changing the parameters of the segmentation method will produce results with different levels of sensitivity and therefore sweep the 


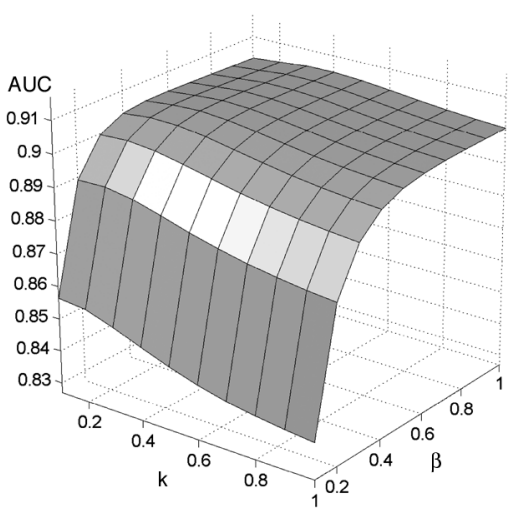

Fig. 4. Parameter optimization for $\beta$ and $k$. The optimal values are $\beta=0.6$ and $k=0.3$.

whole curve. Given a ground truth segmentation, an ideal result will be a point on the upper left corner with the coordinate $(0,1)$. The area under curve (AUC) of ROC curves, which is between 0 and 1 , is considered to be a good measure to quantify ROC curve. The larger the AUC is, the better the method is.

ROC curves have also been used by different researchers [3], [18] to evaluate enhancement performances. Global thresholding is applied to the enhanced images with different thresholds, to obtain segmentations of different levels of sensitivity. AUC of ROC curves can reflect how good an enhancement method is.

Twenty images from the training set of DRIVE are used in this optimization process. For $\beta=\{0.1,0.2, \ldots, 1\}$ and $k=\{0.1,0.2, \ldots, 1\}$, each parameter combination is used in Frangi's measure to enhance 20 images. Once the images are enhanced and normalized, they are thresholded to produce a ROC curve. Only pixels in FOV are considered in ROC analysis. Also, it should be noted that TPR and FPR are calculated based upon all 20 images.

Ten scales with $\sigma_{\min }=1, \sigma_{\max }=5$ are considered, which cover the range of vessel radii in the database. $\gamma=1$ for numerical differentiation.

The results are plotted in Fig. 4. The optimal parameter settings are $\beta=0.6$ and $k=0.3$, hence, $c=$ $0.3 \max \{S(x) \mid x \in \Omega\}$. The corresponding AUC is 0.9055 . However, one can observe that performances around the optimal point are comparable with a slight difference of less than $0.5 \%(0.552 \%$ of the optimal value). This implies the qualitative comparisons based upon the default parameters are still valid in the previous sections.

\section{VARIATIONAL OPTIMIZATION}

In Section III, VE - LLI, an attempt towards a more robust and reliable vesselness measure has been made, in which vessels are modeled as straight line segments. VE - LLI is developed based upon the belief that a good vesselness measure should reflect not only whether a point belongs to a vessel, but also whether the vessel segment is strong or not. It focuses more on the vessel segment to which a point belongs instead of the point itself. For a vessel point, VE - LLI essentially uses a straight line passing through the point as an abstraction of the vessel segment to which the point belongs. However, the use of straight lines and the fact that the probing line has to pass through the point being analyzed can be restrictive. To relax these restrictions, we can consider a better representation of a vessel segment, which is its centerline and not necessary a straight line.

By extracting the centerlines and designing measures based upon the extracted centerlines, some additional advantages can be introduced. Since signals are usually clear and definite along the centerlines, measures based upon centerlines will be more accurate. The use of centerlines in the measure can reduce the level of sensitivity to local structures, due to the fact that the centerline does not have to be close to the particular point under analysis. Moreover, the near-by points belonging to the same vessel segment will have similar vesselness responses after applying the variational optimization (VO) since they share the same centerline. This will give smooth vesselness responses. Furthermore, the narrowing effect of Frangi's measure can be effectively reduced. This is because, for those points inside vessels and near the boundaries, the information used to calculate the measure is collected from the centerline, not local structures.

The VO framework is proposed in this section. The framework takes the enhanced image and its corresponding vessel direction estimations obtained from VE - LLI as inputs and generates optimized vesselness measures. The extended method is called vessel enhancement using local line integrals and variational optimization (VE - LLI - VO). It embeds a more generic vessel model and calculates an optimized vesselness measure based upon features of the possible vessel segment (more specifically, the centerline of the vessel segment) to which a point belongs, in order to enhance vessels with a larger and better view.

\section{A. Optimization Functional}

Given a preliminary enhancement result $E(\mathbf{x})$ and its corresponding vessel direction estimations $\vec{V}(\mathbf{x})$, it is desirable to locate the vessel segment in which a point resides, if there is any, and take information obtained from this vessel segment to develop a measure. In order to achieve this, an efficient and elegant representation of a vessel segment is needed. The centerline of a vessel segment is such a good representation because large vesselness responses can usually be observed along the vessel centerlines. Following this observation, for any point $\mathbf{x}$, an open curve $C_{0}(s)$, which passes through $\mathbf{x}$ will be initialized and let evolve. The goal is to place the curve such that it passes through the centerline of the vessel segment to which $\mathbf{x}$ belongs, if there is any. The final curve $C^{*}(s)$ will have large vesselness responses along it and its tangent vectors should coincide with vessel directions $\vec{V}(\mathbf{x})$. Notice that the final curve $C^{*}(s)$ does not necessarily pass through the point $\mathbf{x}$ as $C_{0}(s)$ does. Therefore, $C^{*}(s)$ depends less upon the point itself, but more on the vessel segment to which the point belongs. The following functional is proposed so that curves passing through vessel centerlines have large values

$$
P(C)=\int_{0}^{L}|\langle\vec{T}(C(s)), \vec{t}(s)\rangle| d s
$$


where $s$ is the arc-length parameter of $C(s)$ and $L$ is the length of $C(s) \cdot \vec{T}=E \vec{V}$

$$
\vec{t}(s)=\frac{d C(s)}{d s}
$$

is the unit tangent vector of curve $C(s)$. Note that $|\vec{t}(s)|=1$, since $s$ is the arc-length parameter. This functional is designed to indicate how well $C(s)$ is locally tracking an vessel. The inner product in (13) can be written as

$$
\langle\vec{T}(C(s)), \vec{t}(s)\rangle=\langle E \vec{V}, \vec{t}(s)\rangle=E \cos \theta
$$

where $\theta$ is the angle between the tangent vector and the estimated vessel direction. Thus, the inner product of $\vec{T}$ with the tangent vector $\vec{t}$ should give large value and this indicates a large vesselness response $E$ and good alignment between the estimated vessel direction $\vec{V}$ and the tangent vector $\vec{t}$ (i.e., $\theta$ is small).

While directly maximizing the functional as stated in (13) leads to longer curves, a functional of the form

$$
Y(C)=\frac{P(C)}{L}
$$

can be used to produce curves that have large average $|\langle\vec{T}(C(s)), \vec{t}(s)\rangle|$ values. Since (13) is equivalent to

$$
P(C)=\int_{0}^{L}|\langle\vec{N}(C(s)), \vec{n}(s)\rangle| d s
$$

where $\vec{N}$ is obtained by rotating $\vec{T} 90^{\circ}$ counter-clockwise and $\vec{n}=C_{s s} /\left|C_{s s}\right|$ is the unit normal direction of $C(s)$.

Functionals of similar forms as stated in (13) and (16) have been studied first in the context of vessel segmentation by Vasilevskiy and Siddiqi [12]. While only closed curves are considered in [12], Kimmel and Bruckstein [19] later proposed an open curve model for such functionals and derived evolution equations for open curves. Inspired by the work done by Kimmel and Bruckstein [19], the evolution equations for our functional $Y(C)$ are

$$
C_{t}=\left(\operatorname{sign}(\langle\vec{N}, \vec{n}\rangle)(\nabla \cdot \vec{N})+\frac{P}{L} \kappa\right) \vec{n}
$$

along the curve and

$$
\begin{aligned}
C_{t}(0) & =(P-L\langle\vec{N}, \vec{n}\rangle) \vec{t}+\operatorname{sign}(\langle\vec{N}, \vec{n}\rangle)\langle\vec{N}, \vec{t}\rangle \vec{n} \\
C_{t}(L) & =(L\langle\vec{N}, \vec{n}\rangle-P) \vec{t}-\operatorname{sign}(\langle\vec{N}, \vec{n}\rangle)\langle\vec{N}, \vec{t}\rangle \vec{n}
\end{aligned}
$$

for end points, where $C_{t}$ can be understood as the moving directions of points on $C . \kappa=\left|C_{s s}\right|$. Derivation of (18)-(20) are given in the Appendix.

The evolution of the curve is based upon the gradient descent rule with respect to the cost functional $Y(C)$. The curve evolution tries to maximize the local vesselness responses and consistencies between its tangent vectors and the estimated vessel directions, while imposing smoothness constraint through the

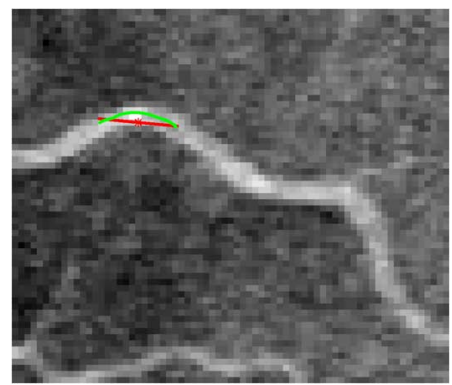

Fig. 5. (Color images) Initialized curve $C_{0}(s)$, shown in red, with their corresponding final position $C^{*}(s)$ shown in green.

curvature $\kappa . Y\left(C^{*}(s)\right)$ of the converged curve will be a good indicator on how strong the possible vessel is. If a point $\mathbf{x}$ is inside the background, the initialized curve will hardly move and the final $Y$ will be small. On the other hand, if $\mathbf{x}$ belongs to a vessel segment, the curve is likely to move to the centerline of the vessel segment. Therefore, we design our new optimized vesselness measure to be $Y\left(C^{*}(s)\right)$.

\section{B. Implementation}

1) Initialization: A good initialization of curve $C(s)$ will not only reduce the time it takes until convergence is reached, but also will guarantee quality of the final curve. Since vessel direction estimations are available, the initial curve at $\mathbf{x}$ can be defined as a straight line, in the vessel direction centering at $\mathbf{x}$

$$
C_{0}(s)=\mathbf{x}+\left(s-\frac{L_{0}}{2}\right) \vec{V}(\mathbf{x})
$$

where $s$ is the arc-length parameter ranging from 0 to $L_{0}, \mathbf{x}$ is the point to be enhanced and $L_{0}$ is the length of the initial line.

\section{Discretization}

A curve $C(s)$ with length $L$ is discretized into $2 L+1$ sample points. That is to say, points are sampled off the curve with $s=\{0,0.5,1, \ldots, L\}$. The time increment $\Delta t$ for the evolution (18), (19) and (20) is empirically set to 0.02 , in order to make a compromise between numerical stability and convergence time.

1) Re-Parameterization: In order to maintain numerical stability, $\Delta t$ should be small. Also, being the arc-length parameter, $s$ needs to cover the curve uniformly. Therefore, a reparametrization is needed after a certain number of iterations. The number of iterations between two reparametrizations, $\Delta i$, is empirically set to 100 , hereinafter. Reparametrization is performed by first calculating the length $L$ of the current curve $C(s) . L$ is approximated by summing up the distances between the neighboring sample points of $C(s)$. Then these sample points are regarded as control points for a quadratic (order 3 ) B-spline. $2 L+1$ new points are then sampled off this spline with equal distances.

2) Other Issues: Curves will stop evolving when the average displacement of points falls below a threshold $\epsilon$. Also, the evolution will stop once the overall length of the curve exceeds $L_{\max }$, to avoid over-smoothing and pathological cases. $\epsilon$ is chosen as 0.001 . As for $L_{\max }$, given the optimal value of $d$ in VE - LLI for a certain dataset, $L_{0}$ and $L_{\max }$ can be set to 

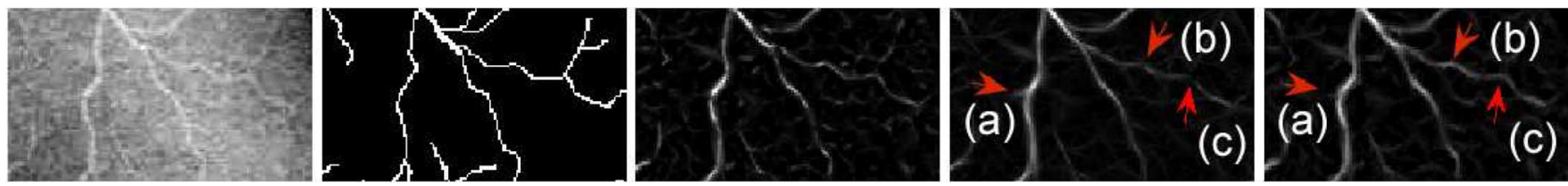

Fig. 6. (Color images) Comparison of enhancement results. From left to right: the original image, its ground truth, enhancement results obtained by using the Frangi's method, VE - LLI and VE - LLI - VO. Red arrows point to regions of interest that show differences between VE - LLI and VE - LLI - VO
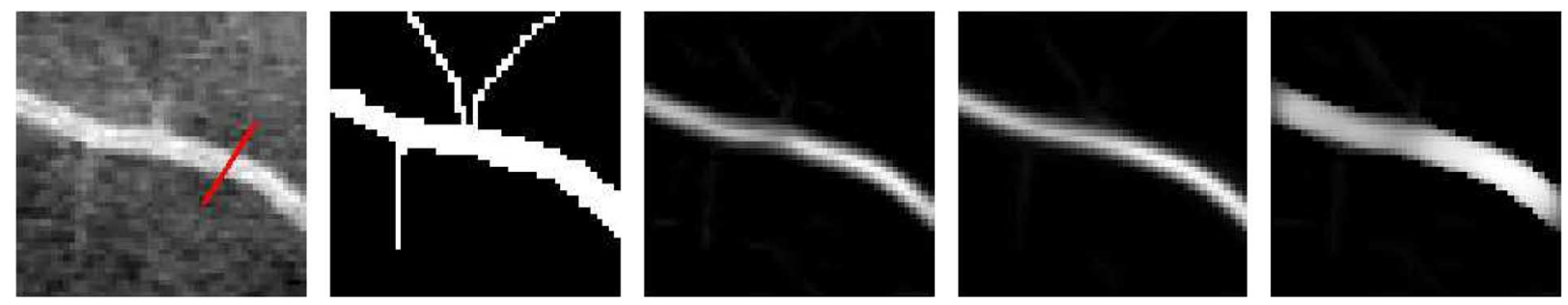

Fig. 7. (Color images) Comparison of enhancement results. From left to right: the original image, its ground truth, enhancement results obtained by using the Frangi's method, VE - LLI and VE - LLI - VO, respectively. The red line in the leftmost figure indicates the cross section of the vessel segment.

satisfy $L_{0}=d<L_{\max }$, to ensure that the length of the final curve approximates $d$. Empirically, $L_{\max }$ is set to $\lceil 1.2 d\rceil$.

\section{Performance Analysis}

One of the advantages of using a variational framework for locating vessels is that the final position of the centerline-searching curve does not necessarily pass through the point being analyzed. It stops at a place where the functional $Y(C)$ is maximized. Therefore, curves initialized at near-by points belonging to the same vessel segment will have similar final positions and, hence, give similar optimized vesselness measures. For curves initialized at background points, they will stop after several iterations near the original position, due to the lack of image forces. This advantage will effectively reduce the narrowing effect on the images, since the optimized vesselness response will depend upon the common vessel centerline. Furthermore, VE - LLI - VO allows more flexibility than VE - LLI. VE - LLI - VO can capture the locations of vessels even when they are not straight. The previously shown properties are further confirmed by experiments and comparisons.

In order to show how VE - LLI - VO works, initial curve, together with its final positions obtained by using VE - LLI - VO is shown in Fig. 5. An image obtained from DRIVE was used for this illustration. Ten scales were considered with $\sigma_{\min }=1$ and $\sigma_{\max }=5$. VE $-\mathbf{L L I}$ was first applied to this image with the optimal parameter settings to obtain preliminary vesselness responses and vessel direction estimations. Then VO was applied on the input with default parameters. The value of $d$ was set to 13 empirically for DRIVE, thus, $L_{0}=13$ and $L_{\max }=16$. The region of interest was selected containing sinuous vessel. Note that, this is a place where a line segment cannot represent the vessel. In this figure, initial curve is shown in red, with the point at consideration shown as an asterisk in the middle, while the final curve is shown in green. It can be observed that final position of the curve is located near the centerline and it can effectively capture the location of the vessel segment.
Moreover, vesselness responses optimized by VE - LLI - VO have been compared with the results obtained by using the Frangi's measure and VE - LLI alone. Comparisons are shown in Figs. 6 and 7. In Fig. 6, the enhancement results of a low contrast region containing vessels are shown. VE - LLI - VO and VE - LLI give comparable results that are both better than the results obtained by using the Frangi's method. They can both produce smooth vesselness measures while suppressing background noise. However, by comparing details in the regions pointed by red arrows, one can see artifacts in the results obtained by using VE - LLI. Points off the vessel are enhanced in region (a). Small sinuous vessels lose their original shape and have low responses in regions (b) and (c). This is caused by the use of straight line segments as the vessel model. On the other hand, VE - LLI - VO, with a more flexible curve model, does not have similar artifacts and produces satisfactory responses. Artifacts from VE-LLI will likely cause problems for applications targeting vessels in low-contrast regions, in which vessels are usually small, sinuous and with low vessel-background contrast. In Fig. 7, another set of vessel segment enhancement results are shown. It can be observed that VE - LLI - VO produces responses that are the closest to the ground truth.

\section{EXPERIMENTS}

Experiments have been conducted on synthetic images and the two retinal image databases, DRIVE and STARE, to evaluate the performance of the new vessel enhancement method. Comparisons have also been made between Frangi's method and VE - LLI - VO, and the experimental results will be presented in this section.

\section{A. Synthetic Images Experiment}

The new method has been evaluated with a 2-D synthetic image containing various vascular structures with additive Gaussian noise (zero mean, 0.01 variance), as it is depicted in Fig. 8. Ten scales were used with $\sigma_{\min }=1$ and $\sigma_{\max }=5$. Since the synthetic image was not retinal image, the default 


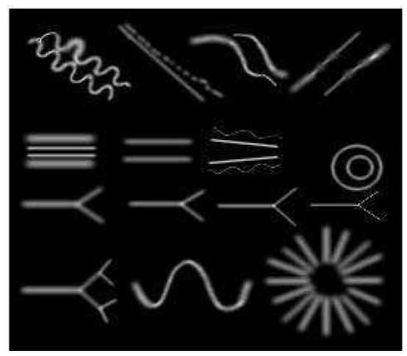

(a)

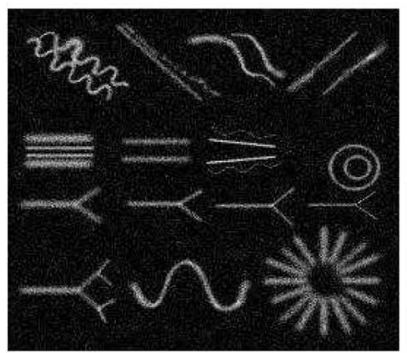

(b)

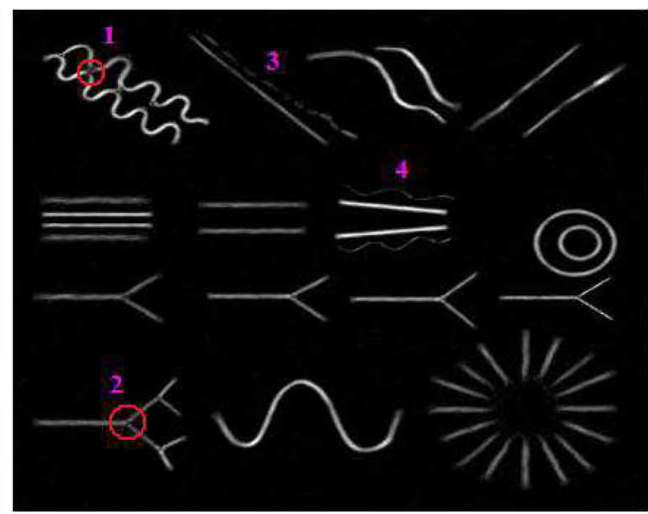

(c)

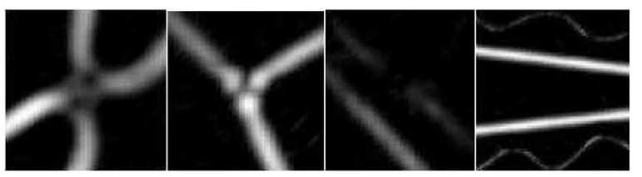

(c. 1) (c. 2) (c.4)

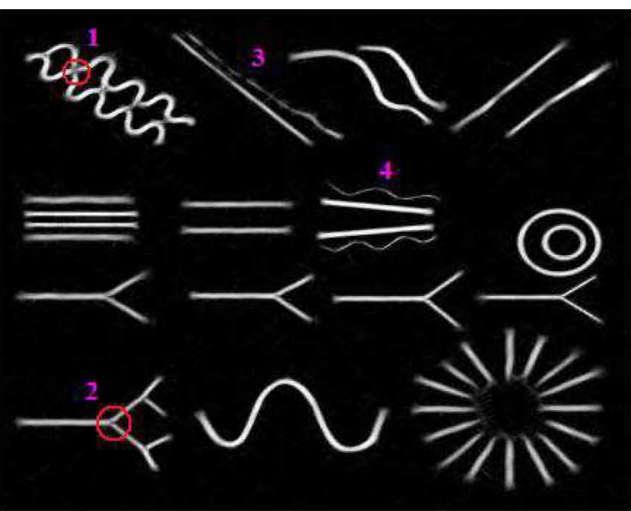

(d)

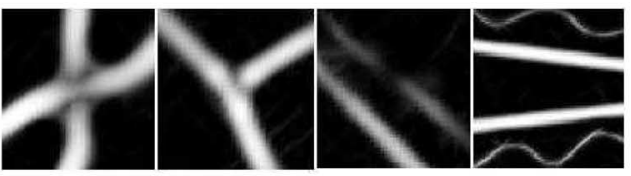

(d.1) (d.2) (d.4)

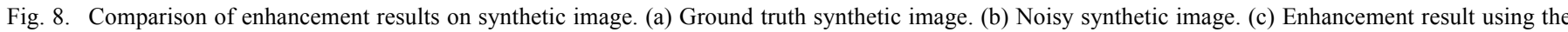
Frangi's method and the closeups of indexed regions. (d) Enhancement result using VE - LLI - VO method and the closeups of indexed regions.

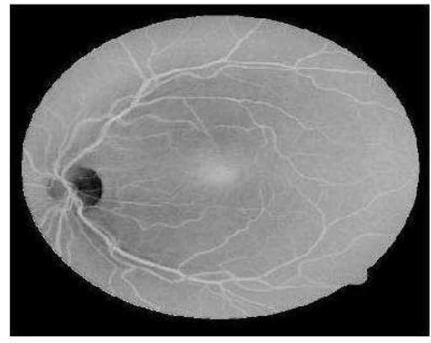

(a)

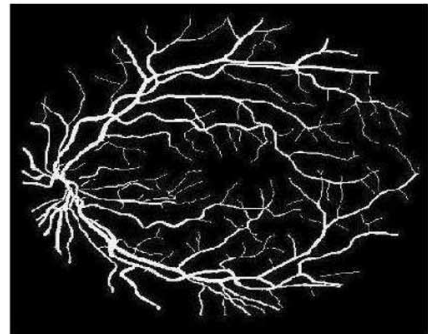

(b)

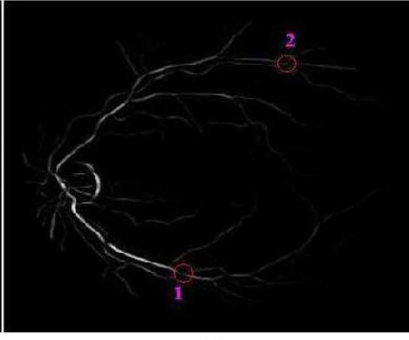

(c)

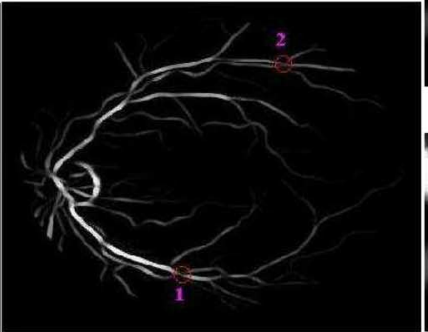

(d)

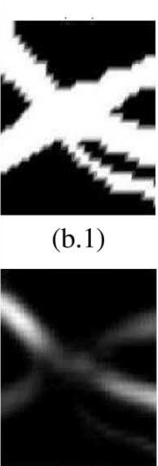

(c.1)

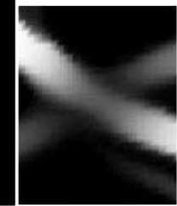

(d.1)

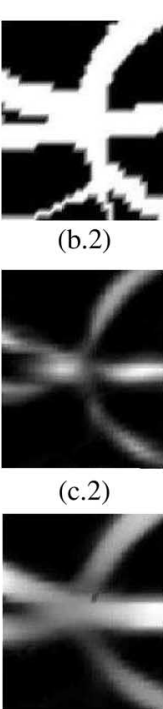

(d.2)

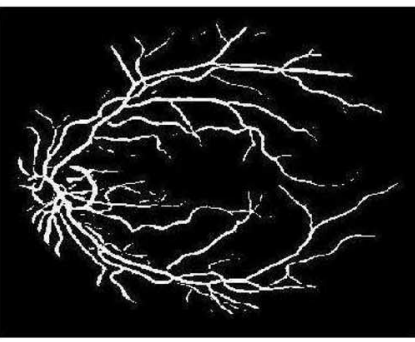

(e)

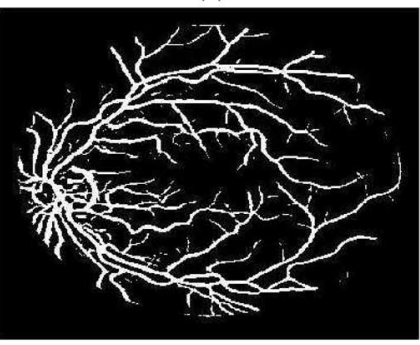

(f)

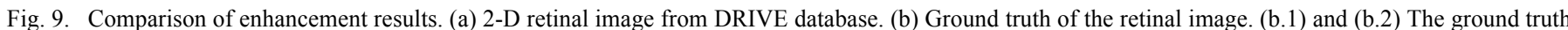

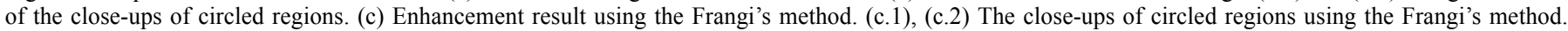

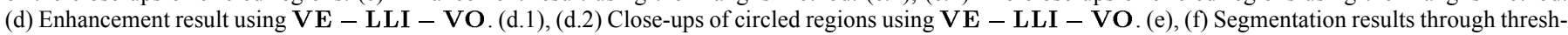
olding the enhancement results in (c) and (d), respectively.

parameter settings for Frangi's measure were used in the experiments. For VE - LLI - VO, preliminary vesselness measures and vessel direction estimations obtained by using VE - LLI were used as inputs. $\Delta t=0.02, \Delta i=100$, $\epsilon=0.001, L_{0}=7$ and $L_{\max }=9$. It is observed from the comparison results, see Fig. 8(c) for Frangi's method and Fig. 8(d) for VE - LLI - VO, that VE - LLI - VO can help give better enhancement results. With close-up comparisons of several junction regions and high curvature structures (see Fig. 8(c.1)-8(c.3), and Fig. 8(d.1)-8(d.3)), VE - LLI - VO obviously can help solve the limitations of the original Hessian-based method.

\section{B. Retinal Images Experiments}

In this section, experiments have been conducted on both DRIVE and STARE databases. Enhancement results obtained by using the Frangi's method and VE - LLI - VO are compared qualitatively in Fig. 9.

To quantitatively evaluate the results, two performance measures were used, namely, angular discrepancy (AD) and area under curve (AUC) of ROC curves. Let $\Omega$ be the image domain and $\Omega_{v} \subseteq \Omega$ be the vessel regions, given the ground truth of an image

$$
I^{*}(\mathbf{x})= \begin{cases}1, & \text { if } \mathbf{x} \in \Omega_{v} \\ 0, & \text { otherwise }\end{cases}
$$




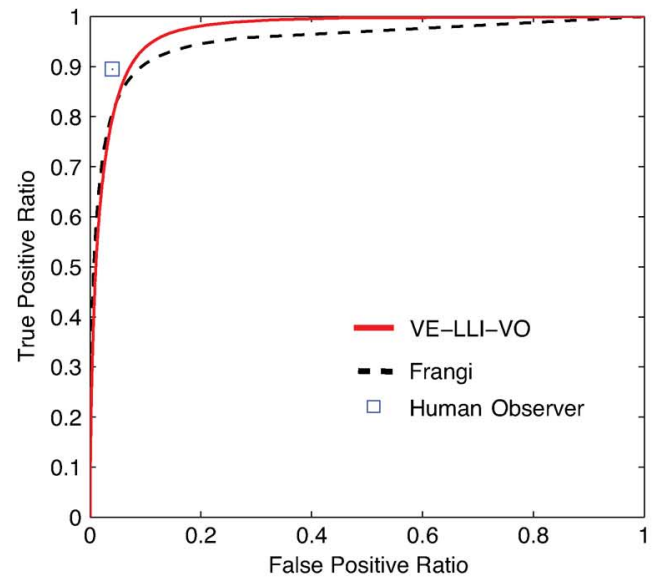

(a)

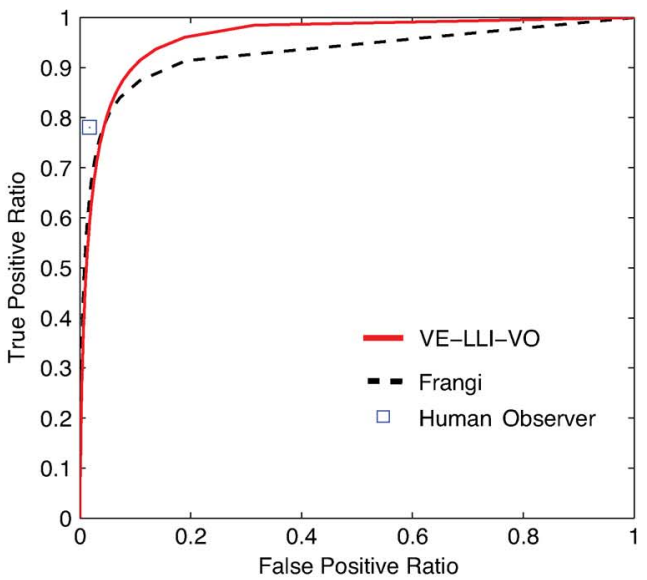

(b)

Fig. 10. (Color images) ROC curves of different methods for (a) DRIVE and (b) STARE, together with the segmented results from the second observer.

TABLE II

SUMMARY OF ANGULAR DISCREPANCIES FOR DRIVE AND STARE. AVERAGE VALUES ARE Listed FOR EACH METHOD With VARIANCES

\begin{tabular}{|c|c|c|}
\hline & $\begin{array}{c}\text { DRIVE } \\
\text { Average AD (variance) }\end{array}$ & $\begin{array}{c}\text { STARE } \\
\text { Average AD (variance) }\end{array}$ \\
\hline \hline Frangi's & $0.1916\left(4.49 \times 10^{-4}\right)$ & $0.1598\left(5.58 \times 10^{-4}\right)$ \\
VE-LLI-VO & $0.1775\left(3.88 \times 10^{-4}\right)$ & $0.1475\left(3.57 \times 10^{-4}\right)$ \\
\hline
\end{tabular}

and its corresponding vessel directions

$$
\vec{V}^{*}: \Omega_{v} \rightarrow \mathbb{R}^{2}
$$

an angular discrepancy can be defined as

$$
A D=\frac{1}{N_{v}} \sum_{\mathbf{x} \in \Omega_{v}}\left|\left\langle\vec{V}(\mathbf{x}),\left[\begin{array}{cc}
0 & -1 \\
1 & 0
\end{array}\right] \vec{V}^{*}(\mathbf{x})\right\rangle\right|
$$

where $N_{v}=\left|\Omega_{v}\right|$ and $\vec{V}(\mathbf{x})$ is the estimated vessel direction at point $\mathbf{x}$. The inner product of $\vec{V}(\mathbf{x})$ with the normal to $\vec{V}^{*}(\mathbf{x})$ is proportional to $\sin \theta$, where $\theta$ is the angle between $\vec{V}(\mathbf{x})$ and $\vec{V}^{*}(\mathbf{x})$. A smaller AD means a better estimation of vessel directions.

Since neither of the two databases at consideration provides vessel directions associated with the ground truth, an estimation of vessel directions $\vec{V}^{*}(\mathbf{x})$ can be obtained by applying the Frangi's method on the ground truth $I^{*}(\mathbf{x})$. Ten scales were used with $\sigma_{\min }=1$ and $\sigma_{\max }=5$ for DRIVE. Fourteen scales were considered for STARE with $\sigma_{\min }=1$ and $\sigma_{\max }=8 . \beta=0.6$ and $c=0.3 \max \{S(\mathbf{x}) \mid \mathbf{x} \in \Omega\} . d_{D}=13$ for DRIVE database and $d_{S}=21$ for STARE database. Default parameters were used in VO.

First, angular discrepancies have been evaluated for each image in the two databases using the two methods. The results are summarized in Table II. It can be observed that VE - LLI - VO has a smaller angular discrepancy than the Frangi's method in both databases. This is because VE - LLI - VO uses a different principle for estimating vessel directions. In the Frangi's method, vessel directions are all estimated based upon eigenvectors of the Hessian matrix; while in VE - LLI - VO, it is achieved by considering local line integrals of the Hessian matrix.
TABLE III

AVERAge AUCs AND VARIANCES For INDIVIDUAL IMAGeS IN DRIVE AND STARE

\begin{tabular}{|c|c|c|}
\hline & DRIVE & STARE \\
& Average AUC (variance) & Average AUC (variance) \\
\hline \hline Frangi's & $0.9321\left(3.27 \times 10^{-4}\right)$ & $0.9518\left(3.37 \times 10^{-4}\right)$ \\
VE-LLI-VO & $0.9641\left(0.74 \times 10^{-4}\right)$ & $0.9715\left(2.42 \times 10^{-4}\right)$ \\
Jiang et al. & $0.9327(\mathrm{~N} / \mathrm{A})$ & $0.9298(\mathrm{~N} / \mathrm{A})$ \\
Staal et al. & 0.9520 (N/A) & 0.9614 (N/A) \\
\hline
\end{tabular}

Second, ROC analysis has been applied to individual images in the two databases. AUCs were recorded for each image. The average ROC curves for the two database are given in Fig. 10. For the DRIVE database, the manual segmentations from the first set were used as the ground truth and the manual segmentations of the second set were used to indicate the human observer results, which provided only one FPR-TPR pair shown as blue square in Fig. 10. For the STARE database, the first observer's manual segmentations were used as the ground truth and the second observer's results were indicated as the human observer results. Average AUCs and variances of the Frangi's method and VE - LLI - VO are summarized in Table III, together with the results obtained by Jiang et al. and Staal et al., as these results have been published in [15]. It is observed that VE - LLI - VO can give better enhancement results in the retinal images. Both VE - LLI - VO and Frangi's method were implemented in the MATLAB environment. The computation time of VE - LLI - VO, VE - LLI and Frangi's method were respectively around $4 \mathrm{~h}, 30$ mins and 10 mins for each retinal image on a PC with a Pentium IV $3.2 \mathrm{GHz}$ CPU and 1 GB RAM. The computation time can be further reduced by using the graphical processing units (GPUs).

\section{CONCLUSION}

Contributions of this work can be summarized as follows. In this paper, limitations of the Hessian-based vessel enhancement methods have been discussed, which stem from the fact that the Hessian matrix is a local measure sensitive to local intensity structures. A new view on the vesselness measure has been proposed. A more robust vesselness measure should not only reflect 
whether a point belongs to a vessel segment, but also whether the vessel segment is a strong one or not. The new view on the vesselness measure shifts focus from a point to the vessel segment to which the point belongs.

Vessel enhancement using local line integrals (VE - LLI) is first proposed in this paper. A vessel model is embedded as the prior knowledge by regarding a vessel segment as a straight line and using the second order information of the local line integrals. VE - LLI is less sensitive to local intensity abnormalities. Therefore, it can produce more accurate vesselness measures and vessel direction estimations. Useful quantities similar to the eigenvalues and eigenvectors of the Hessian matrix are produced by VE - LLI. These quantities can be exploited in any existing Hessian-based vesselness measure methods. With the local line integrals, junction regions can be detected and handled effectively with smaller line integral length. This can give better enhancement results than the existing Hessian-based methods which will suppress these junction regions. A variational optimization (VO) framework is further proposed to improve quality of vesselness measures. It is used as an extension on VE - LLI to form VE - LLI - VO. VE - LLI - VO can produce smooth vesselness responses along vessels, give good performance in background suppression, and reduce the narrowing effect of some Hessian-based methods. VE - LLI - VO utilizes a more generic curve model instead of straight line segments used in VE - LLI. Therefore, it can locate vessels with large curvatures, which is a limitation for VE - LLI.

\section{APPENDIX}

The objective is to maximize

$$
Y(C)=\frac{P(C)}{L}
$$

with

$$
P(C)=\int_{0}^{L}|\langle\vec{N}(C(s)), \vec{n}(s)\rangle| d s
$$

which measures both the vesselness response along the curve and the degree of alignment between the tangent vector of the curve and the estimated vessel direction.

First, let

$$
L(C)=\int_{0}^{1}\left|C_{p}\right| d p
$$

be the arclength of an open curve $C_{p}$. Adding the variation $\eta(p)$ to the curve, such that $\widetilde{C}(p)=C(p)+\epsilon \eta(p)$, differentiating w.r.t. $\epsilon$ and letting $\epsilon$ go to zero, yields

$$
L^{\prime}(C)=-\int_{0}^{L} \kappa\langle\vec{n}, \eta\rangle d s+\langle\eta(L), \vec{t}(L)\rangle-\langle\eta(0), \vec{t}(0)\rangle
$$

where $\mathrm{s}$ is the arclength parameter and $\kappa=\left|C_{s s}\right| . P(C)$ can be rewritten as a general form

$$
P(C)=\int_{0}^{L} \rho(\langle\vec{N}, \vec{n}\rangle) d s .
$$

with $\rho(\alpha)=|\alpha|$. After taking the first variation

$$
P^{\prime}(C)=\int_{0}^{L} \eta \frac{\partial Y}{\partial C} d s+\left.\eta\left(\rho \vec{t}-\rho^{\prime}\langle\vec{N}, \vec{t}\rangle \vec{n}\right)\right|_{0} ^{L} .
$$

Using this derived condition in the Fua-Leclerc formula [19] yields along the curve

$$
L \frac{\partial Y}{\partial C}+P \kappa \vec{n}=0
$$

and

$$
L\left(\rho \vec{t}-\rho^{\prime}\langle\vec{N}, \vec{t}\rangle \vec{n}\right)=P \vec{t}
$$

at the end points $C(L)$ and $C(0)$.

We use these conditions to guide a gradient descent process for an active contour evolution toward the local maximum of $Y(C)$. For $\rho(\alpha)=|\alpha|$ in our case, the gradient descent flow along the curve is given by

$$
C_{t}=\left(\operatorname{sign}(\langle\vec{N}, \vec{n}\rangle)(\nabla \cdot \vec{N})+\frac{P}{L} \kappa\right) \vec{n}
$$

along the curve and

$$
\begin{aligned}
C_{t}(0) & =(P-L\langle\vec{N}, \vec{n}\rangle) \vec{t}+\operatorname{sign}(\langle\vec{N}, \vec{n}\rangle)\langle\vec{N}, \vec{t}\rangle \vec{n} \\
C_{t}(L) & =(L\langle\vec{N}, \vec{n}\rangle-P) \vec{t}-\operatorname{sign}(\langle\vec{N}, \vec{n}\rangle)\langle\vec{N}, \vec{t}\rangle \vec{n}
\end{aligned}
$$

for end points, where $C_{t}$ can be understood as the moving directions of points on $C$. The motion of the end point is designed in the way that it either extends or shrinks the curve so that it satisfies the constraint in (A8).

\section{REFERENCES}

[1] T. Koller, G. Gerig, G. Szekely, and D. Dettwiler, "Multiscale detection of curvilinear structures in 2-D and 3-D image data," in Proc. Int. Conf. Comput. Vis., 1995, pp. 864-869.

[2] K. Krissian, G. Malandain, N. Ayache, R. Vaillant, and Y. Trousset, "Model based multiscale detection of 3-D vessels," in Proc. Comput. Vis. Pattern Recognit., 1998, pp. 722-727.

[3] Y. Sato, S. Nakajima, N. Shiraga, H. Atsumi, S. Yoshida, T. Koller, G. Gerig, and R. Kikinis, "Three-dimensional multi-scale line filter for segmentation and visualization of curvilinear structures in medical images," Med. Image Anal., vol. 2, no. 2, pp. 143-168, 1998.

[4] A. Frangi, W. Niessen, K. Vincken, and M. Viergever, "Multiscale vessel enhancement filtering," in Proc. Med. Image Comput. Comput.Assist. Intervent., 1998, vol. 1496, pp. 130-137.

[5] Q. Li, S. Sone, and K. Doi, "Selective enhancement filters for nodules, vessels, and airway walls in two-and three-dimensional CT scans," Med. Phys., vol. 30, pp. 20-40, 2003.

[6] C. Lorenz, I. Carlsen, T. Buzug, C. Fassnacht, and J. Weese, "Multiscale line segmentation with automatic estimation of width, contrast and tangential direction in 2-D and 3-D medical images," in Proc. 1st Joint Conf. Comput. Vis. Virtual Reality Robot. Med. Med. Robot. Comput.-Assist. Surgery, 1997, vol. 1205, pp. 233-242. 
[7] C. Lorenz, I. Carlsen, T. Buzug, and C. Fassnacht, "A multi-scale line filter with automatic scale selection based on the hessian matrix for medical image segmentation," in Proc. 1st Int. Conf. Scale-Space Theory Comput. Vis., 1997, vol. 1252, pp. 152-163.

[8] J. Hladůvka and E. Gröller, "Exploiting eigenvalues of the Hessian matrix for volume decimation," in Proc. Int. Conf. Central Eur. Comput. Graph., Visual. Comput. Vis., 2001, pp. 124-129.

[9] B. Morse, S. Pizer, and A. Liu, "Multiscale medial analysis of medical images," Image Vis. Comput., vol. 12, no. 6, pp. 327-338, 1994.

[10] R. Manniesing and W. Niessen, "Multiscale vessel enhancing diffusion in CT angiography noise filtering," in Proc. Inf. Process. Med. Imag., 2005 , vol. 3565 , pp. $138-149$.

[11] C. Cańero and P. Radeva, "Vesselness enhancement diffusion," Pattern Recognit. Lett., vol. 24, no. 16, pp. 3141-3151, 2003.

[12] A. Vasilevskiy and K. Siddiqi, "Flux maximizing geometric flows," IEEE Trans. Pattern Anal. Mach. Intell., vol. 24, no. 12, pp. 1565-1578, Dec. 2002.

[13] T. Lindeberg, "Edge detection and ridge detection with automatic scale selection," Int. J. Comput. Vis., vol. 30, no. 2, pp. 117-154, 1998.

[14] O. Tom, An Introduction to Signal Processing in Chemical Analysis. College Park: Univ. Maryland Press, 2009 [Online]. Available: http://terpconnect.umd.edu/ toh/spectrum/PeakFindingandMeasurement.htm

[15] J. Staal, M. Abramoff, M. Niemeijer, M. Viergever, and B. van Ginneken, "Ridge-based vessel segmentation in color images of the retina," IEEE Trans. Med. Imag., vol. 23, no. 4, pp. 501-509, Apr. 2004.

[16] M. Niemeijer, J. Staal, B. van Ginneken, M. Loog, and M. Abramoff, "Comparative study of retinal vessel segmentation methods on a new publicly available database," SPIE Med. Imag., vol. 5370, pp. 648-656, 2004.

[17] A. Hoover, V. Kouznetsova, and M. Goldbaum, "Locating blood vessels in retinal images by piecewise thresholdprobing of a matched filter response," IEEE Trans. Med. Imag., vol. 19, no. 3, pp. 203-210, Mar. 2000.

[18] B. Chapman and D. Parker, "3-D multi-scale vessel enhancement filtering based on curvature measurements: Application to time-of-flight MRA,” Med. Image Anal., vol. 9, no. 3, pp. 191-208, 2005.

[19] R. Kimmel and A. Bruckstein, "Regularized laplacian zero crossings as optimal edge integrators," Int. J. Comput. Vis., vol. 53, no. 3, pp. 225-243, 2003.

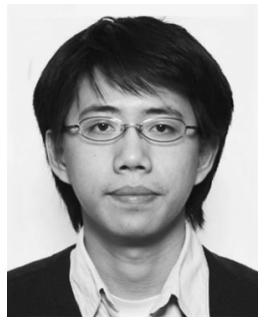

Yuan Yuan received the B.Sc. degree in mathematics and M.Phil. degree in computer science and engineering from The Hong Kong University of Science and Technology, Hong Kong, in 2006 and 2008 , respectively.

$\mathrm{He}$ is currently with the Lo Kwee-Seong Medical Image Analysis Laboratory, Department of Computer Science and Engineering, The Hong Kong University of Science and Technology, Hong Kong. His research interests include medical image processing and analysis, vessel enhancement, and

segmentation

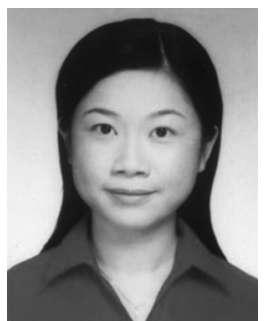

Yishan Luo received the B.Sc. and M.Sc. degrees in electronic engineering from Fudan University, Shanghai, China, in 2005 and 2008, respectively, and is currently pursuing the Ph.D. degree in computer science and engineering at The Hong Kong University of Science and Technology, Hong Kong.

Her research interests include medical image registration and segmentation, image enhancement.

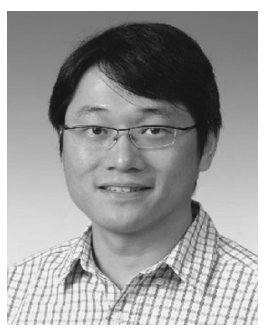

Albert C. S. Chung received the B.Eng. degree (first class honors) in computer engineering from The University of Hong Kong in 1995 and the M.Phil. degree in computer science from The Hong Kong University of Science and Technology in 1998.

He joined the Medical Vision Laboratory, University of Oxford, Oxford, U.K., as a Doctoral Research Student with a Croucher Foundation scholarship and graduated in 2001. He was a Visiting Scientist at the Artificial Intelligence Laboratory, Massachusetts Institute of Technology, Cambridge, in 2001. He is currently an Associate Professor with the Department of Computer Science and Engineering, The Hong Kong University of Science and Technology. His research interests include medical image analysis, image processing, and computer vision.

Dr. Chung won the 2002 British Machine Vision Association Sullivan Thesis Award for the best doctoral thesis submitted to a U.K. university in the field of computer or natural vision. 